\title{
Self-Image and Suicide in a Swedish National Eating Disorders Clinical Register
}

\author{
Cristin D. Runfola, Ph.D. ${ }^{a}$, Laura M. Thornton, Ph.D. ${ }^{a}$, Emily M. Pisetsky, Ph.D. ${ }^{b}$, Cynthia M. \\ Bulik, Ph.D. ${ }^{a, c}$, and Andreas Birgegård, Ph.D. ${ }^{d}$ \\ a Department of Psychiatry, University of North Carolina, Chapel Hill, NC \\ b Department of Psychology, University of North Carolina, Chapel Hill, NC \\ c Department of Nutrition, University of North Carolina, Chapel Hill, NC \\ d Department of Clinical Neuroscience, Karolinska Institutet, Stockholm, Sweden
}

\begin{abstract}
Objective-Using a prospective design, to examine the relation between self-image (assessed using the Structural Analysis of Social Behavior) and suicide attempts/completions in women with anorexia nervosa-restricting type (ANR), anorexia nervosa-binge/purge type (ANBP), bulimia nervosa (BN), binge eating disorder, and eating disorder not otherwise specified (EDNOS); and to assess whether these self-image variables add unique predictive value to suicide when considering other baseline predictors.
\end{abstract}

Method-Women $(N=2,269)$ age 12 to $45(M=22.1)$ presenting to specialist eating disorders clinics in Sweden between 2005 and 2009 were identified through the Stepwise Eating Disorders Quality Register. Data on age, body mass index, eating disorder severity (Eating Disorder Examination-Questionnaire scores), psychiatric comorbidity, global assessment of functioning, and self-image were abstracted from Stepwise and included as baseline predictors or covariates. Suicide information (prior attempt and attempt/completion after Stepwise registration) was obtained from the National Patient Register and Cause of Death Register.

Results-Prevalence of detected suicide attempts/completions over the study period was $9.2 \%$. Negative self-image variables were associated with prior suicide attempts in ANR and EDNOS and later suicide attempts/completions in women with BN. In a stepwise Cox proportional hazards model, only low self-affirmation predicted time to suicide attempts/completions in women with $\mathrm{BN}$ when accounting for age and prior suicide attempt.

Conclusion-Assessing self-image might assist with identifying women with $\mathrm{BN}$ at elevated risk for suicide.

\section{Keywords}

SASB; self-image; suicide; eating disorders; anorexia; bulimia; binge eating disorder

\footnotetext{
(c) 2013 Elsevier Inc. All rights reserved.

Correspondence: Cynthia M. Bulik, PhD, Department of Psychiatry, University of North Carolina at Chapel Hill, CB \#7160, 101 Manning Drive, Chapel Hill, NC 27599-7160, USA. Voice: (919) 843-1689; Fax: (919) 842-8802; cbulik@ med.unc.edu..

Publisher's Disclaimer: This is a PDF file of an unedited manuscript that has been accepted for publication. As a service to our customers we are providing this early version of the manuscript. The manuscript will undergo copyediting, typesetting, and review of the resulting proof before it is published in its final citable form. Please note that during the production process errors may be discovered which could affect the content, and all legal disclaimers that apply to the journal pertain.
} 


\section{Introduction}

Risk of suicide is dramatically elevated in individuals with eating disorders. The standardized mortality ratio (SMR) for suicide is between 31.0 and 32.4 for anorexia nervosa (AN) [1,2] and 7.5 and 30.9 for bulimia nervosa (BN) [2,3]. Suicide attempts, one of the best predictors of suicide completion [4], are also frequent, severe, and often characterized by strong intent to die, high lethality, and high medical threat in individuals with AN and BN [5-7]. Multiple correlates of suicide attempts in individuals with eating disorders have been identified, including purging, depression, substance abuse [8], anxiety [5,9-11], impulse-control disturbances $[5,6,12,13]$, cluster $\mathrm{B}$ and $\mathrm{C}$ personality disorders $[5,9,11]$, and certain temperament and character traits as measured by the Temperament and Character Inventory [14], such as high persistence, self-transcendence [15], and harm avoidance [5,9,12], and low self-directedness [5,12,15]. However, developing reliable suicide risk profiles for patients with eating disorders is imperative to improve vigilance, detection, and prevention of destructive behaviors and death in this high-risk population.

A potential risk factor for suicide, not yet examined in eating disorder patients, is self-image. Broadly defined, self-image refers to how one views or conceives of oneself, including one's perception of personal attributes, worth, competencies, and coping resources [16]. Selfimage, specifically self-blame, has been correlated with suicidal behaviors in other psychiatric populations [17,18], and it (e.g., self-affirmation) relates to personal characteristics, such as receptiveness and defensiveness [19] that may impact responsiveness to therapy, such as the receipt of health information [20-23]. The Structural Analysis of Social Behavior (SASB) $[20,24,25]$ is one assessment for evaluating self-image that has been applied in eating disorders research to improve understanding of treatment adherence [26], treatment satisfaction [27], and clinical outcome [28,29]. The SASB is advantageous because it yields scores (ranging from high to low) on eight variables (self-emancipation, self-affirmation, self-love, self-protection, self-control, self-blame, self-hate, and selfneglect) that collectively provide detailed information on the respondent's self-perception and typical self-regulatory style. Rather than more static self-worth or descriptive personal attributes, the SASB codifies self-treatment, i.e., how a person behaves toward him/herself, in terms of orthogonal affiliative (friendly vs. hostile) and emancipatory (control vs. let go) dimensions in a circumplex model, with the eight variables as the end points and their diagonal combinations. Further, SASB self-image is empirically grounded in interpersonal theory [30] and provides clinically meaningful information. For example, SASB self-image is hypothesized [30] to reflect the respondent's interpersonal expectancies or perceptual biases that shape his/her behavior and evoke complementary responses in others, ultimately perpetuating interpersonal patterns and reinforcing his/her self-image (i.e., self-fulfilling prophecy). To our knowledge, no other such clinically relevant and widely applicable [31] measure of self-image exists.

Moreover, SASB scores can help predict future behavior [31], including the potential for parasuicidal acts (according to a small study of patients with borderline personality disorder) [32]. In a clinical eating disorder sample, SASB self-image variables differentiated individuals who dropped out of treatment (e.g., less self-blame) from those who completed treatment [26] and uniquely predicted domain-specific and global outcome independent of other baseline clinical symptoms [28,29]. Negative self-image profiles predicting poor global outcome were high self-control in those with AN and high self-hate and low self-love in those with BN [29]. Importantly, SASB self-image is amenable to treatment [33]. Thus, identifying the relation between SASB self-image and suicide attempts/completions might inform not only risk assessment, but also suicide prevention in this population. 
The primary aims of our study were: 1) to explore whether SASB self-image, assessed at initial presentation, is associated with prior suicide attempts in women diagnosed with AN restricting type (ANR), AN binge-eating/purging type (ANBP), BN, binge eating disorder (BED), and eating disorder not otherwise specified (EDNOS); 2) using a prospective design, to identify whether baseline self-image predicts time to subsequent suicide attempts/ completions in each diagnostic group; and 3) to examine whether SASB variables identified in aim 2 add unique predictive value to time to subsequent suicide attempts/completions in individuals with ANR, ANBP, BN, and EDNOS when considering other baseline predictors. All analyses were exploratory.

\section{Methods}

Swedish women with an eating disorder diagnosed between 2005 and 2009 were identified through the Swedish quality assurance database, Stepwise (see below) [34]. History of suicide attempts as well as suicide attempt and completion data following initial entry into Stepwise (i.e., initial presentation to an eating disorders clinic) were obtained from The Swedish National Patient Register [35] and Swedish Cause of Death Register [36]. These datasets were linked using the National Registration Number [37], a unique personal identifier assigned to all Swedish citizens. The Research Ethics Committee of the Karolinska Institutet approved data collection procedures, and The University of North Carolina at Chapel Hill Institutional Review Board determined that analysis of the de-identified data was exempt.

\section{Description of Swedish Registers}

Stepwise-Stepwise [34] is a longitudinal internet-based quality assurance register, developed in 2003, capturing nearly all inpatient and outpatient specialized eating disorder care in Sweden since 2005. Over 20 specialized eating disorders centers participated in Stepwise at the time of data extraction, providing good coverage. Stepwise contains comprehensive eating disorder-specific and general psychiatric information on all patients. A clinical psychologist, psychiatrist, psychiatric nurse, or social worker with specialty training (completion of a 2-day mandatory course) in the Stepwise-based assessment of eating disorders conduct and facilitate all testing. Assessment takes place on initial presentation (baseline) to the clinic once intent to treat is established (usually within 3 visits for outpatients and 1 week for inpatients) and quarterly thereafter. The present study only uses data obtained from baseline testing. Assessment results from every patient are entered into Stepwise for clinical purposes but consent is required to use the information for research. About 3\% of registrants decline participation in research. Patients who declined were significantly younger (but with a less than small effect size; Cohen's $d<0.20$ ) than those who agreed to participate, and patients with AN were slightly more likely to decline than patients with $\mathrm{BN}$, although the association was weak (Cramer's $V<0.10$ ). There was no significant association between declining participation in research and time of entry into the database. Stepwise development, methodology, and data quality are described in detail elsewhere [34].

National Patient Register-The National Patient Register [35] is an epidemiologic population-based dataset established in 1964 that covers all public and private hospital admissions in Sweden. Full coverage began in 1987 for inpatient admissions and in 2001 for outpatient admissions [38]. We had access to data through 2009. Primary discharge diagnosis and up to eight secondary diagnoses are captured using codes from the International Classification of Diseases, Ninth Revision (ICD-9; 1987-1996) [39] or International Classification of Diseases, Tenth Revision (ICD-10; 1997-present) [40]. Suicide attempts are routinely coded. Validity checks demonstrate high reliability with 
regard to accurate coding of diagnoses [41,42], and a recent review documented a positive predictive value (PPV) of between $85-95 \%$ for inpatient diagnoses [43].

The Cause of Death Register-The Cause of Death Register [36] contains information on age and cause of death (based on ICD codes) abstracted from the death certificates of all Swedish registrants since 1952 . We had access to data through 2008. Only individuals who died in Sweden and were registered in Sweden at the time of death are included in this register. Death by suicide is coded as such. The database has excellent coverage (includes $99.7 \%$ of all deaths), and reliability of official cause-of-death statistics for diagnoses appears high [44].

\section{Study Sample}

All patients registered in Stepwise who met the following criteria were included in the study: 1) a DSM-IV eating disorder diagnosis (ANR, ANBP, BN, BED, or EDNOS) [45]; 2) female sex; 3) 12-45 years of age at initial entry into Stepwise (because early- and late-age onset eating disorders may differ meaningfully from typical age at onset cases); 4) initial entry into Stepwise between 2005 and 2009; and 5) complete SASB data.

The total dataset included 3,040 patients after removing individuals with no eating disorder diagnosis and selecting the first clinic visit for those with multiple visits up through 2009. Patients were also excluded from analyses for the following not mutually exclusive reasons: no SASB information $(n=65)$, male sex $(n=96)$, age $>45$ years at registration $(n=64)$, and EDNOS diagnosis of "chewing and spitting" (only identified in those whose initial entry was after February 2008; $n=21$ ). Given that depression is an established risk factor for suicide [46] and has been strongly and consistently correlated with suicide attempts in patients with eating disorders [8], all individuals with missing information on lifetime history of depression $(n=600)$ were excluded from analyses. Data for a total of 2,248 women were available for analysis.

\section{Eating Disorder Diagnosis}

Eating disorders were diagnosed by specially trained clinicians using semi-structured clinical interviews [for adults, the Structured Clinical Interview for DSM-IV Axis I Disorders, SCID-I [47], and, for children and adolescents, the Mini International Neuropsychiatric Interview, M.I.N.I.Kid [48,49], were used until February 2008; the Structured Eating Disorder Interview, SEDI [50], was used thereafter], expert clinician judgment, and a structured process for verifying and re-classifying (if necessary) SEDIbased non-eating disorder diagnoses (see Birgegård, Norring, \& Clinton [51] for a thorough description of diagnostic procedures). Clinicians coded diagnoses based on DSM-IV [45] classification criteria as follows: ANR (AN with no regular binge-eating or purging), ANBP (AN with regular binge-eating or purging), BN, BED, and EDNOS. EDNOS consisted of subthreshold AN (35.6\%) and subthreshold BN (16.1\% non-purging type; 48.4\% purgingtype or purging disorder).

\section{Suicide Attempts/Completions}

Information on suicide attempts was extracted from the National Patient Register (years 1973-2009) and identified by ICD codes to capture definite (ICD-9: E950-E959; ICD-10: X60-X84) and uncertain cases (ICD-9: E980-989; ICD-10: Y10-34) [39,40]. Information about death by suicide was obtained from the Cause of Death Register (years 2005-2008 for this study), identified by ICD codes to also capture definite (ICD-9: E950-E959; ICD-10: X60-X84) and uncertain cases (ICD-9: E980-E989; ICD-10: Y10-Y34). Some uncertain cases are in fact deaths by suicide [52,53], but cases classified as either definite or uncertain do differ on some parameters such as age, method used, and presence of a suicide note [54]. 
The exclusion of uncertain cases may underestimate the true number of suicides but including all uncertain cases may conversely exaggerate suicide rates and result in falsepositive misclassification. Thus, in this study, we created two variables to capture 1) definite cases and 2) only uncertain cases so that we could compare these groups on characteristics of the suicide attempts/completion to provide an empirically supported rationale for including/excluding uncertain cases in analyses.

\section{SASB Self-image}

Self-image was examined using the SASB, $3^{\text {rd }}$ surface, Introject $[20,24,25]$ self-report questionnaire, which yields a well-validated [30] circumplex model providing detailed information about self-regulatory style (i.e., how one treats or regards oneself) across two dimensions-affiliation (degree of self-love/self-rejection on vertical axis) and interdependence (degree of self-emancipation/self-control on horizontal axis). The Introject circumplex figure can be viewed in Birgegård et al. [29]. It includes 36 self-referential statements (framed either positively or negatively) that are rated on a scale of 0-100 in 10point increments depending on the degree to which the item reflects accurately the respondent's self-regulatory style $(0=$ not at all, $100=$ completely). Three example items are as follows: 1) "Without considering what might happen, I hatefully reject and destroy myself." (self-hate); 2) "I put energy into providing for, looking after, developing myself." (selfprotection); and 3) "Knowing both my faults and strong points, I comfortably let myself be as is." (self-affirmation) A rating of 40 or above indicates confirmation of the item. The survey yields eight cluster scores (i.e., self-image variables), each comprising four or five items: self-emancipation, self-affirmation, self-love, self-protection, self-control, self-blame, self-hate, and self-neglect. The cluster scores are the means of the items comprising the cluster (sum of cluster item scores / number of items in cluster). High values ( $\geq 40$ ) on the positive clusters and low values ( $\$ 40$ ) on the negative clusters characterize a healthy selfimage. The SASB self-image has demonstrated reliability with the current Stepwise sample (mean alpha coefficient=.75). It is applied broadly in therapeutic settings to assess, educate, and help direct and assess patient interventions [31].

\section{Baseline Characteristics}

All baseline characteristics were abstracted from the Stepwise assessment and are detailed below.

Demographics-Age and body mass index (BMI) at initial presentation were recorded. BMI was calculated from height and weight $\left(B M I=\mathrm{kg} / \mathrm{m}^{2}\right)$, which were either self-reported or measured by clinic staff using standard anthropometric procedures. The proportion of patients with measured weight by diagnosis was as follows (data from January 2010 August 2012: $\mathrm{AN}=80.8 \%, \mathrm{BN}=56.7 \%, \mathrm{EDNOS}=62.3 \%$ (total=64.7\%). The BMI of patients with measured weight was significantly lower than the BMI of patients with self-reported weight in AN, $t(775)=-2.40, p=.016, d=-0.22$, and EDNOS, $t(836)=-6.27, p=.001, d=$ -0.33 , but not $\mathrm{BN}, t(836)=-0.76, p=.44, d=-0.05$, and effect sizes were small.

Eating disorder severity-The Eating Disorder Examination-Questionnaire (EDE-Q) [55] was used to index current eating disorder symptom severity. A modified version of the EDE-Q [56], with simplified language and a shortened time frame (14- instead of 28-days), was used for adolescents ( $<18$ years). The EDE-Q is comprised of 36 items, each scored on a seven-point scale ranging from 0 (no days/not at all) to 6 (every day/markedly), and yields four subscales measuring restraint, eating concern, shape concern, and weight concern, and a global score (average of all subscales). Higher scores are indicative of greater severity. Reliability [57] and validity [58] for the EDE-Q have been established. 
Psychiatric disorders-Trained clinical interviewers diagnosed comorbid psychiatric disorders (current/lifetime) using the SCID-I [47] for adults (ages $\geq 18$ ) and M.I.N.I.Kid $[48,49]$ for youth $($ ages $<18)$. Although different in format, the SCID-I and M.I.N.I.Kid are both semi-structured clinical interviews that provide comparable outcome data: DSM-IV psychiatric diagnoses. Psychiatric diagnoses evaluated in this study included lifetime 1) depression (major depressive episode, major depressive disorder), 2) anxiety (generalized anxiety disorder, social anxiety, panic disorder, agoraphobia, specific phobia, obsessivecompulsive disorder, anxiety disorder NOS, or separation anxiety disorder), and 3) posttraumatic stress disorder. Current alcohol abuse/dependence and substance abuse/ dependence (stimulants, sedatives, cannabis, cocaine, opiates, hallucinogens, other, or polysubstance abuse or dependence) were also evaluated.

General psychopathology-The DSM global assessment of functioning (GAF) rating scale was used as a measure of current (day of evaluation) psychosocial functioning [45]. The GAF accounts for both psychiatric symptom severity and impairment in social and occupational functioning. Scores range from 1-100 (91-100 = superior functioning and no symptoms). Although the validity and reliability of the GAF have been questioned [59], GAF ratings have been shown to predict suicide attempts in various clinical populations [60,61], including AN [62].

\section{Statistical Analyses}

All statistical analyses were conducted using SAS ${ }^{\circledR}$ version 9.2 [63]. Descriptive statistics and graphics were used to screen data for implausible values, errors, outliers, and potential influential observations, and to check distributional assumptions. To determine whether to include uncertain cases in our outcome variable of suicide attempts/completions, we compared individuals with at least one definite suicide attempt/completion to individuals with only uncertain suicide attempts/completions on age at first attempt and violence of attempts ('violent' vs. 'non-violent'). Although groups did not differ in mean age of first attempt, $t(24.7)=1.94, p=.07$, women with at least one definite suicide attempt/completion were significantly more likely to have had at least one violent attempt than women with only uncertain suicide attempts/completions $(29.6 \%$ vs. $5.6 \%), \chi^{2}=6.24, p=.013$. These results suggest that it may be inappropriate to combine definite with uncertain suicide cases in analyses. As a result, our primary outcome variable of suicide attempts/completions included only definite cases.

Logistic regression models were applied to examine the association between SASB selfimage variables (self-emancipation, self-affirmation, self-love, self-protection, self-control, self-blame, self-hate, and self-neglect) and prior suicide attempts stratified by eating disorder diagnosis (ANR, ANBP, BN, BED, and EDNOS; aim 1). Cox proportional hazards models were applied to assess whether the eight SASB self-image variables predicted time (in months) to subsequent suicide attempts/completions after registration for ANR, ANBP, BN, and EDNOS (aim 2; BED was not included because the prevalence of suicide attempts/ completions was too low for meaningful comparisons). The Cox regression model allows for unequal follow-up time of participants and includes information from individuals whose suicide attempt status is unknown $[64,65]$. The hazard ratio in the Cox model is similar to a relative risk and refers to the likelihood of an outcome - suicide attempts. Because there is an established association between suicide attempts/completions and age [66], age at baseline was included in all models as a covariate. In addition, previous suicide attempt was included as a covariate in models predicting suicide attempts/completions after registration. We controlled for multiple comparisons using the method of false discovery rate (FDR) [67]. A $p$-value $<.05$ was considered statistically significant. 
To evaluate whether significant SASB variables identified in aim 2 added unique predictive value to suicide attempts/completions after registration, we first applied Cox proportional hazards models to determine significant associations between each of the baseline characteristics (BMI, the four EDE-Q subscales, depression, anxiety, post-traumatic stress disorder, alcohol and substance abuse/dependent, and GAF) and suicide attempts/ completions for each diagnostic group (ANR, ANBP, BN, and EDNOS). We checked for multicollinearity (with no issues found) and then applied a backward stepwise Cox model predicting time to suicide attempts/completions from only those SASB variables and baseline characteristics found to be significant in prior models. Age at baseline and prior suicide attempt were entered and retained in all models. The probability to leave the model was set at .05 .

\section{Results \\ Sample Characteristics}

The mean (SD) age of our sample was 22.1 (7.0) years. In the total sample, $206(9.2 \%)$ women had at least one suicide attempt/completion during the study period (Table 1). Of these women, 140 ( $6.2 \%$ of the total sample) had at least one suicide attempt prior to registration into Stepwise and 86 (3.8\% of the total sample) had at least one suicide attempt/ completion (two deaths) after registration. The average length of follow-up was 25.83 months (minimum $=0.10$, maximum $=58.70 ; \mathrm{SD}=17.33$ ). The deaths occurred in one woman with ANR (violent attempt) and one woman with EDNOS (non-violent attempt), both of whom had no prior suicide attempt. The prevalence of suicide attempts prior to registration was significantly higher in women with BN than in women with ANR, $\chi^{2}(4)=6.24, p=.013$, and EDNOS, $\chi^{2}(4)=10.30, p=.002$. In contrast, the prevalence of suicide attempts/ completions after registration was significantly higher in women with ANBP than in women with any other eating disorder diagnosis ( $p<.018$ for all pair-wise comparisons with ANBP); there were no other significant differences between eating disorder diagnoses.

The means $(S D)$ of SASB variables by presence of suicide attempts/completions occurring prior to and after registration for each eating disorder diagnosis are presented in Tables 2 and 3, respectively. As can be seen in both tables, all eating disorder diagnoses were characterized by negative self-image regardless of suicide attempt/completion history. Low values $(\leq 40)$ on the positive clusters and high values $(\geq 40)$ on the negative cluster indicate negative self-image.

\section{Association Between SASB Self-image Variables and Prior Suicide Attempts}

Table 4 presents results of logistic regression analyses evaluating the association between SASB variables and prior suicide attempts for each eating disorder diagnosis (aim 1). After controlling for age and correcting for multiple comparisons, results revealed that prior suicide attempt was significantly associated with 1) lower self-affirmation, lower self-love, higher self-blame, higher self-hate, and higher self-neglect in women with ANR; 2) higher self-affirmation in women with $\mathrm{BN}$; and 3) lower self-love, lower self-protection, higher self-blame, higher self-hate, and higher self-neglect in women with EDNOS. No other statistically significant associations were observed but other clinically interesting findings emerged. Effect size estimates showed that individuals with ANBP who had past suicide attempts had lower self-emancipation $(d=0.73)$, self-affirmation $(d=0.88)$, and self-love $(d=1.11)$, and higher self-blame ( $d=-0.58)$ than those with ANBP with no past attempts. In addition, individuals with BED who had past suicide attempts had lower self-affirmation $(d=0.83)$, self-love $(d=1.01)$, and self-protection $(d=0.71)$, and higher self-blame $(d=-0.56)$, self-hate $(d=-0.63)$, and self-neglect $(d=-0.58)$ than those with BED with no past suicide attempts. 


\section{SASB Self-image Variables as Predictors of Time to Suicide Attempts/Completions}

Table 5 presents results for analyses assessing whether SASB variables predict time to subsequent suicide attempts/completions after registration (aim 2). Hazard ratios greater than 1 indicate a higher probability of suicide attempts whereas ratios less than 1 indicate a lower probability of attempts. Thus, lower self-affirmation and self-love, and higher selfblame, self-hate, and self-neglect significantly predicted time to suicide attempts/ completions in women with BN when controlling for age and prior suicide attempt. No other statistically significant associations were observed.

Since significant associations between the SASB variables and time to suicide attempts/ completions after registration were found only in those with $\mathrm{BN}$, analyses for aim 3 were only applied to this diagnostic subgroup. For BN, the baseline characteristics lifetime anxiety diagnosis $\left(\chi^{2}=4.24, p=.040\right)$, GAF score $\left(\chi^{2}=7.14, p=.008\right)$, and the EDE-Q restraint $\left(\chi^{2}=5.17, p=.023\right)$, weight concern $\left(\chi^{2}=4.89, p=.027\right)$, and shape concern $\left(\chi^{2}=5.25, p=.022\right)$ subscales were significantly associated with time to suicide attempts/completions after registration. When entered into the final stepwise Cox model along with self-affirmation, self-love, self-blame, self-hate, and self-neglect, only self-affirmation was retained along with the covariates of prior suicide attempt and age.

\section{Discussion}

This is the first study to examine associations between SASB self-image and suicide attempts in a registry-based sample of eating disorder patients, and is one of the largest studies to date to identify prospective predictors of time to suicide attempts/completions in eating disorder patients by diagnosis and subtype (ANR, ANBP, BN, EDNOS). Our results shed light on which patients who enter treatment might be at increased risk for suicide and suggest potential clinical utility of assessing self-image in patients at presentation.

The prevalence of lifetime suicide attempts/completions in our total sample of $9.2 \%$ is lower than that $(25 \%)$ reported in another heterogeneous eating disorder sample [11]. Variation in suicide assessment methods (self-report vs. medically detected cases) [68] and sample characteristics (e.g., inclusion of some non-treatment seeking individuals vs. clinical-only sample; and slightly older sample with mean age of 29 years vs. 22 years) may account for this difference. After intake, 3.8\% of our sample had at least one detected suicide attempt/ completion. Studies with longer follow-up periods yield higher prevalence of suicide attempts in individuals with eating disorders (e.g., 15\% prevalence within 8.6 years) [62] suggesting compounding risk over time. Compared with the general population, the lifetime prevalence of suicide attempts in eating disorder patients is high [69], underscoring need for specialized risk protocols for this group.

Eating disorders characterized by binge eating and purging (BN, ANBP) vs. restricting (ANR) had the highest prevalence of prior suicide attempts at intake, corroborating prior reports [5,11,13,70-72]. Also, significantly more women in the ANBP group had attempted/ completed suicide after presenting for treatment than in any other group. The prevalence of suicide attempts in the ANBP group after intake (10.2\%) was notably higher than before presentation (7.8\%), raising an important and unexpected observation of increased rather than decreased risk of suicide after presenting for treatment in individuals with ANBP. Reasons for this finding are unknown but warrant further exploration. As the prevalence of suicide attempts in BED was the lowest of all diagnostic groups, it may be the presence of purging (not binge eating) that is most strongly associated with suicidal behavior; this observation has some empirical support $[8,11]$. 
Consistent with another study [73], all eating disorder diagnoses were characterized by negative self-image. Low self-love and high self-blame were associated with prior suicide attempts across all disorders (except BN). Self-blame, assessed using other measures, has been correlated previously with suicidal behaviors in sexual assault survivors [17] and individuals with depression [18]. Self-blame is also a primary motive recorded in the suicide notes of individuals who completed suicide [74]. These results are unsurprising and have considerable face validity; individuals high in self-blame likely internalize and personalize negative interactions or life experiences, and with little buffering self-love, may be inclined to use impulsive drastic measures to escape these negative emotions or to "self-punish" [75].

Intriguingly, in women with $\mathrm{BN}$, prior suicide attempts were related to a higher degree of self-affirmation—on the surface a perplexing result. One could speculate that interventions or responses to the attempt may have positively influenced self-image in this group. According to Sullivan's predictive principle of introjection (treat yourself as did others), which forms the basis of the SASB self-image [31], a patient can become more self-affirmed simply by a therapist's communication of acceptance and affirmation. Perhaps the patients with BN who attempted suicide received affirming, non-shaming responses from providers or others following their attempts, thereby facilitating change in self-image. However, this is purely speculative and requires more detailed investigation to verify. Directionality also cannot be inferred in the cross-sectional analyses, and there likely exists a bidirectional relationship between self-image and suicide. Further, multiple other negative SASB selfimage variables were related to prior suicide attempt in women with ANR and EDNOS, and several medium-to large effect sizes were also observed in women with ANBP and BED. Generally, these results suggest that a past history of a suicide attempt is related to negative self-image in all eating disorders except BN.

Our results also suggest SASB may assist with predicting a future suicide attempt in patients with BN. In our univariate analyses, low self-affirmation and self-love, and high self-blame, self-hate, and self-neglect in BN predicted time to suicide attempts after registration even when accounting for age and prior suicide attempt. However, in the final model, only low self-affirmation proved predictive of suicide attempts in women with BN. It is understandable how someone low in self-affirmation who has an overall negative self-image might be at increased risk for a suicide attempt. People who are self-affirmed are open to new ideas, carefully examine the evidence for their beliefs (rather than fall prey to unhealthy defense mechanisms and cognitive distortions) [19], and likely evoke more positive interactions with others [20]. In clinical studies, self-affirmation has been found to increase responsiveness to threatening health information (i.e., psychoeducation) $[21,22]$ and to promote health behavior change [23]. Finally, a self-affirmed individual positively accepts the self "as is" [31].

The fact that the relation between self-affirmation and self-image was opposite depending on whether the attempt was in the past versus future may lend support to our above hypothesis regarding a bidirectional relationship between the two variables. Studies examining the mechanisms underlying these relations are needed to clarify associations.

Many of the baseline characteristics that failed to predict future suicide attempts/ completions in the prospective portion of the study have been associated with suicide attempts in previous cross-sectional investigations (i.e., lifetime depression [5,6,9,10,15]; lifetime substance abuse/dependence $[5,6,9,13,71])$. These discrepancies underscore the importance of interpreting correlational studies with caution and highlight the need for additional well-powered prospective reports to ascertain true risk factors for suicide. Our findings are fairly consistent with a prospective study of suicide in individuals with AN and $\mathrm{BN}$ [62]. However, that study found that lifetime substance abuse predicted later suicide 
attempt in patients with $\mathrm{BN}$, whereas current substance abuse was not predictive of suicide attempts in our investigation.

\section{Clinical Implications}

SASB might be one cost-effective [29] assessment measure that can contribute clinically to suicide risk assessment in individuals with eating disorders. The benefit of the SASB is that it can also assist the provider (regardless of orientation) with overall case conceptualization [31]. SASB provides information on the patient's general view of him/herself and can help the provider understand the impact of these beliefs on the patient's behaviors and interactions with others that serve to preserve the self-image of that individual [20]. SASB results can be presented to patients in an attempt to increase insight into their behavior and to direct therapeutic interventions. For example, based on the self-fulfilling prophecy and theory of complementarity [20,25], individuals high in self-blame may readily blame themselves when struggling in treatment (e.g., having a slip; not completing homework) and provoke or perceive blaming responses from providers. Interventions focused on exploring patient reactions to therapy, thoughts about setbacks, and beliefs about the therapists' thoughts and reactions to the patient may bring to light faulty cognitions, enhance the therapeutic relationship, and improve self-image $[25,28]$. Given their relation to suicide attempts, high self-blame, low self-love (for all eating disorders but BN), and low selfaffirmation (for $\mathrm{BN}$ ), may constitute particularly important therapeutic targets. Ultimately, self-image enhancement may improve eating disorders outcome [28,29].

\section{Strengths and Limitations}

Lack of selection biases, use of empirically supported measures and structured diagnostic interviews, prospective data, and complete outcome ascertainment constitute particular strengths of the present study. By linking Swedish quality assurance registers of specialized eating disorder care (Stepwise) with Cause of Death and hospital-based registers, we were able to identify all detected cases of suicide attempts/completions in our sample. However, because this was a clinical sample of Swedish women aged 12-45, results might not generalize to those with eating disorders who are not involved in treatment, to men or older women with eating disorders, or to individuals residing in other countries. Moreover, misclassification of suicide on death certificates occurs [52-54], and the prevalence of detected suicide attempts in our sample may be conservative. As such, results might not generalize to cases of suicide attempts that are not detected by the healthcare system or cases not severe enough to warrant medical attention. Further, we were limited to information documented in the registers. We lacked data on other potential predictors of suicide, such as treatment compliance, personality traits and disorders, and family history of suicide attempt. Additionally, we were unable to account for diagnostic crossover between eating disorder diagnoses, as only partial information on this dimension is available in the dataset. Crossover between eating disorder diagnoses is common, and moving from ANR to ANBP may be predictive of later suicide attempt [76]. Different, although developmentally appropriate, measures were used to ascertain diagnoses across age, and BMI was assessed by varying methods (self-report or measured). Moreover, even though the study was conducted in a high-risk population for suicide attempts, some cell sizes were small, particularly in ANBP and BED groups, and some confidence intervals were wide; Type II errors may have occurred. Further failure to detect significant differences in the EDNOS group may be due to the fact that this is a heterogeneous sample including patients with subthreshold AN, subthreshold BN, and purging only behaviors. Future studies should examine predictors of suicide in these subgroups separately. Lastly, we were only able to address outcome within 3 years after registration; longer follow-ups yield higher prevalence suicide attempts/completions in eating disorders, and provide greater statistical power. 


\section{Conclusions}

Assessing self-image in eating disorders patients might provide clinically relevant information regarding suicide history and potential for future attempts (in those with BN). Lower levels of self-affirmation predict subsequent suicide attempts in individuals with BN. Additional prospective studies with longer follow-up periods are needed to validate findings and identify the most robust predictors of suicide so that better risk assessments can be created and employed clinically. Future studies should also include other potential predictors of suicide such as duration of illness, eating disorder diagnostic crossover, treatment history, treatment compliance, family history of suicide, personality disorders, and TCI personality characteristics.

\section{Acknowledgments}

This study was supported in part by a grant from the American Foundation for Suicide Prevention (PI: Bulik). The Stepwise database is financially supported by Stockholm County Council. Dr. Runfola was supported by the National Institutes of Health (NIH) Grant T32MH076694 (PI: Bulik) and the Global Foundation for Eating Disorders Scholar Program [Voice: (212) 475-4700; Website: http://www.gfed.org/].

\section{References}

1. Harris EC, Barraclough B. Excess mortality of mental disorder. Br J Psychiatry. 1998; 173:11-53. [PubMed: 9850203]

2. Preti A, Rocchi MB, Sisti D, Camboni MV, Miotto P. A comprehensive meta-analysis of the risk of suicide in eating disorders. Acta Psychiatr Scand. 2011; 124:6-17. [PubMed: 21092024]

3. Huas C, Godart N, Caille A, Pham-Scottez A, Foulon C, Divac SM, et al. Mortality and its predictors in severe bulimia nervosa patients. Eur Eat Disord Rev. 2013; 21:15-19. [PubMed: 22528211]

4. Jenkins GR, Hale R, Papanastassiou M, Crawford MJ, Tyrer P. Sucide rate 22 years after parasuicide: Cohort study. BMJ. 2002; 325:1115. [PubMed: 12424180]

5. Bulik CM, Thornton L, Pinheiro AP, Plotnicov K, Klump KL, Brandt H, et al. Suicide attempts in anorexia nervosa. Psychosom Med. 2008; 70:378-383. [PubMed: 18256339]

6. Corcos M, Taïeb O, Benoit-Lamy S, Paterniti S, Jeammet P, Flament MF. Suicide attempts in women with bulimia nervosa: Frequency and characteristics. Acta Psychiatr Scand. 2002; 106:381386. [PubMed: 12366473]

7. Guillaume S, Jaussent I, Olié E, Genty C, Bringer J, Courtet P, et al. Characteristics of suicide attempts in anorexia and bulimia nervosa: A case-control study. PLoS ONE. 2011; 6:e23578. [PubMed: 21858173]

8. Franko DL, Keel PK. Suicidality in eating disorders: Occurrence, correlates, and clinical implications. Clin Psychol Rev. 2006; 26:769-782. [PubMed: 16875766]

9. Anderson CB, Carter FA, McIntosh VV, Joyce PR, Bulik CM. Self-harm and suicide attempts in individuals with bulimia nervosa. EDJ. 2002; 10:227-243.

10. Forcano L, Álvarez E, Santamaría JJ, Jimenez-Murcia S, Granero R, Penelo E, et al. Suicide attempts in anorexia nervosa subtypes. Compr Psychiatry. 2011; 52:352-358. [PubMed: 21683172]

11. Milos G, Spindler A, Hepp U, Schnyder U. Suicide attempts and suicidal ideation: Links with psychiatric comorbidity in eating disorder subjects. Gen Hosp Psychiatry. 2006; 26:129-135. [PubMed: 15038930]

12. Forcano L, Fernández-Aranda F, Alavarez-Moya E, Bulik C, Granero R, Gratacós M, et al. Suicide attempts in bulimia nervosa: Personality and psychopatholocial correlates. European Psychiatry. 2009; 24:91-97. [PubMed: 19101125]

13. Stein D, Lilenfeld RR, Widman PC, Marcus MD. Attempted suicide and self-injury in patients diagnosed with eating disorders. Compr Psychiatry. 2004; 45:447-451. [PubMed: 15526255]

14. Cloninger, CR. The Temperament and Character Inventory (TCI): A guide to its development and use. Center for Psychobiology and Personality, Washington University; St. Louis, MO: 1994. 
15. Bulik CM, Sullivan PF, Joyce PR. Temperament, character and suicide attempts in anorexia nervosa, bulimia nervosa and major depression. Acta Psychiatr Scand. 1999; 100:27-32. [PubMed: 10442436]

16. Baumeister, RF., editor. The Self in Social Psychology. Psychology Press, Taylor \& Francis; Philadelphia, PA: 1999.

17. Ullman SE, Najdowski CJ. Correlates of serious suicidal ideation and attempts in female adult sexual assault survivors. Suicide Life Threat Behav. 2009; 39:47-57. [PubMed: 19298150]

18. Grunebaum MF, Keilp J, Li S, Ellis SP, Burke AK, Oquendo MA, Mann JJ. Symptom components of standard depression scales and past suicidal behavior. J Affect Disord. 2005; 87:73-82. [PubMed: 15923041]

19. Correll J, Spencer SJ, Zanna MP. An affirmed self and an open mind: Self-affirmation and sensitivity to argument strength. J Exp Soc Psychol. 2004; 40:350-356.

20. Benjamin LS. Structural analysis of social behavior. Psychol Rev. 1974; 81:392-425.

21. Sherman DAK, Nelson LD, Steele CM. Do messages about health risks threaten the self? Increasing the acceptance of threatening health messages via self-affirmation. Pers Soc Psychol Bull. 2000; 26:1046-1058.

22. van Koningsbruggen GM, Das E, Roskos-Ewoldsen DR. How self-affirmation reduces defensive processing of threatening health information: Evidence at the implicit level. Health Psychology. 2009; 28:563-568. [PubMed: 19751082]

23. Epton T, Harris PR. Self-affirmation promotes health behavior change. Health Psychology. 2008; 27:746-752. [PubMed: 19025270]

24. Benjamin LS. Use of Structural Analysis of Social Behavior (SASB) and Markov chains to study dyadic interactions. J Abnorm Psychol. 1979; 88:303-319. [PubMed: 500958]

25. Benjamin LS, Rothweiler JC, Critchfield KL. The use of Structural Analysis of Social Behavior (SASB) as an assessment tool. Annu Rev Clin Psychol. 2006; 2:83-109. [PubMed: 17716065]

26. Björck C, Björk T, Clinton D, Sohlberg S, Norring C. Self-image and treatment drop-out in eating disorders. Psychol Psychother. 2008; 81:95-104. [PubMed: 17631699]

27. Björck T, Björck C, Clinton D, Sohlberg S, Norring C. What happened to the ones who dropped out? Outcome in eating disorder patients who complete or prematurely terminate treatment. Eur Eat Disord Rev. 2009; 17:109-119. [PubMed: 19142975]

28. Björck C, Clinton D, Sohlberg S, Norring C. Negative self-image and outcome in eating disorders: Results at 3-year follow-up. Eat Behav. 2007; 8:398-406. [PubMed: 17606238]

29. Birgegård A, Björck C, Norring C, Sohlberg S, Clinton D. Anorexic self-control and bulimic selfhate: Differential outcome prediction from initial self-image. Int J Eat Disord. 2009; 42:522-530. [PubMed: 19107836]

30. Henry WP. Structural analysis of social behavior as a common metric for programmatic psychopathology and psychotherapy research. J Consult Clin Psychol. 1996; 64:1263-1275. [PubMed: 8991313]

31. Benjamin LS. Introduction to the special section on Structural Analysis of Social Behavior. J Consult Clin Psychol. 1996; 64:1203-1212. [PubMed: 8991306]

32. Shearin EN, Linehan MM. Patient-therapist ratings and relationship to progress in dialectical behavioral therapy for borderline personality disorder. Behav Ther. 1992; 23:730-741.

33. Dennhag I, Armelius BA. Baseline training in cognitive and psychodynamic psychotherapy during a psychologist training program. Exploring client outcomes in therapies of one or two semesters. Psychother Res. 2012; 22:515-526. [PubMed: 22468963]

34. Birgegård A, Björck C, Clinton D. Quality assurance of specialised treatment of eating disorders using large-scale internet-based collection systems: Methods, results and lessons learned from designing the Stepwise database. Eur Eat Disord Rev. 2010; 18:251-259. [PubMed: 20589767]

35. National Board of Health and Welfare. [May 10, 2012] The National Patient Register. from http:// www.socialstyrelsen.se/register/halsodataregister/patientregistret/inenglish

36. National Board of Health and Welfare. Causes of Death 2008. National Board of Health and Welfare; Stockholm, Sweden: 2010. 
37. Ludvigsson JF, Otterblad-Olausson P, Pettersson BU, Ekbom A. The Swedish personal identity number: Possibilities and pitfalls in healthcare and medical research. Eur J Epidemiol. 2009; 24:659-667. [PubMed: 19504049]

38. Ludvigsson JF, Sellgren C, Runeson B, Långström N, Lichtenstein P. Increased suicide risk in coeliac disease--a Swedish nationwide cohort study. Digest Liver Dis. 2011; 43:616-622.

39. World Health Organization. International Classification of Diseases, Ninth Revision (ICD-9). World Health Organization; Geneva, Switzerland: 1978.

40. World Health Organization. International Classification of Diseases, Tenth Revision (ICD-10). World Health Organization; Geneva, Switzerland: 1992.

41. Dalman CH, Broms J, Cullberg J, Allebeck P. Young cases of schizophrenia identified in a national inpatient register--are the diagnoses valid? Soc Psychiatry Psychiatr Epidemiol. 2001; 37:527-531. [PubMed: 12395142]

42. Razavi D, Ljung R, Lu Y, Andrén-Sandberg A, Lindblad M. Reliability of acute pancreatitis diagnosis coding in a national patient register: A validation study in Sweden. Pancreatology. 2011; 11:525-532. [PubMed: 22094886]

43. Ludvigsson JF, Andersson E, Ekbom A, Feychting M, Kim JL, Reuterwall C, et al. External review and validation of the Swedish national inpatient register. BMC Public Health. 2011; 11:450-466. [PubMed: 21658213]

44. Fall K, Strömberg F, Rosell J, Adrèn O, Varenhorst E, The South-east Region Prostate Cancer Group. Reliability of death certificances in prostate cancer patients. Scand J Urol Nephrol. 2008; 42:352-357. [PubMed: 18609293]

45. American Psychiatric Association. Diagnostic and statistical manual of mental disorders, 4th edition, text revision (DSM-IV-TR). American Psychiatric Publishing Inc; Washington, DC: 2000.

46. Windfuhr K, Kapur N. Suicide and mental illness: A clinical review of 15 years findings from the UK national confidential inquiry into suicide. Br Med Bull. 2011; 100:101-121. [PubMed: 21948337]

47. First, MB.; Gibbon, M.; Spitzer, RL.; Williams, JB. Structured Clinical Interview for the DSMIV axis I disorders. (Herlofson Swedish Adaptation). Pilgrim Press; Danderyd: 1998.

48. Sheehan DV, Lecrubier Y, Sheehan KH, Amorim P, Janavas J, et al. The Mini-International Neuropsychiatric Interview (M.I.N.I): The development and validation of a structured diagnostic interview for DSM-IV and ICD-10. J Clin Psychiat. 1998; 59:22-23.

49. von Knorring, L.; von Knorring, AL.; Björck, C. MINI International Neuropsychiatric Interview for children and adolescents: Swedish version 2.1. Uppsala University/Karolinska Institutet [In Swedish]; Uppsala/Stockholm: 2003.

50. de Man Lapidoth, J.; Birgegård, A. Validation of the Structured Eating Disorder Interview (SEDI) against the Eating Disorder Examination (EDE). Karolinska Institutet; Stockholm: 2010.

51. Birgegård A, Norring C, Clinton D. DSM-IV versus DSM-5: Implementation of proposed DSM-5 criteria in a large naturalistic database. Int J Eat Disord. 2012; 45:353-361. [PubMed: 22506283]

52. Allebeck P, Allgulander C, Henningsohn L, Jakobsson SW. Causes of death in a cohort of 50,465 young men--validity of recorded suicide as underlying cause of death. Scand J Soc Med. 1991; 19:242-247. [PubMed: 1775959]

53. Neeleman J, Wessely S. Changes in classification of suicide in England and Wales: Time trends and associations with coroner' professional backgrounds. Psychol Med. 1997; 27:467-472. [PubMed: 9089838]

54. Linsley KR, Schapira K, Kelly TP. Open verdict v. suicide -- importance to research. Br J Psychiatry. 2001; 178:465-468. [PubMed: 11331564]

55. Fairburn CG, Beglin SJ. Assessment of eating disorders: Interview or self-report questionnaire? Int J Eat Disord. 1994; 16:363-370. [PubMed: 7866415]

56. Carter JC, Stewart DA, Fairburn CG. Eating disorder examination questionnaire: Norms for young adolescent girls. Behav Res Ther. 2001; 39:625-632. [PubMed: 11341255]

57. Luce KH, Crowther JH. The reliability of the eating disorder examination-self-report questionnaire version (EDE-Q). Int J Eat Disord. 1999; 25:349-351. [PubMed: 10192002] 
58. Mond JM, Hay PJ, Rodgers B, Owen C, Beumont PJ. Validity of the Eating Disorder Examination Questionnaire (EDE-Q) in screening for eating disorders in community samples. Behav Res Ther. 2004; 42:551-567. [PubMed: 15033501]

59. Aas IH. Guidelines for rating Global Assessment of Functioning (GAF). Ann Gen Psychiatry. 2011; 20:10-12.

60. Jia CX, Zhang J. Global functioning and suicide among Chinese rural population aged 15-34 years: A psychological autopsy case-control study. J Forensic Sci. 2012; 57:391-397. [PubMed: 22150171]

61. Robinson J, Cotton S, Conus P, Schimmelmann BG, McGorry P, Lambert M. Prevalence and predictors of suicide attempt in an incidence cohort of 661 young people with first-episode psychosis. Aust N Z J Psychiatry. 2009; 43:149-157. [PubMed: 19153923]

62. Franko DL, Keel PK, Dorer DJ, Blais MA, Delinsky SS, Eddy KT, et al. What predicts suicide attempts in women with eating disorders? Psychol Med. 2004; 34:843-853. [PubMed: 15500305]

63. SAS Institute Inc. SAS/STAT® 9.2 User's Guide. SAS Institute Inc.; Cary, NC: 2008.

64. Bradburn MJ, Clark TG, Love SB, Altman DG. Surival analysis part II: Multivariate data analysis--an introduction to concepts and methods. Br J Cancer. 2003; 89:431-436. [PubMed: 12888808]

65. Collett, D. Modelling survival data in medical research. Chapman \& Hall; London, UK: 1994.

66. Shah A. The relationship between suicide rates and age: An analysis of multinational data from the World Health Organization. Int Psychogeriatr. 2007; 19:1141-1152. [PubMed: 17433118]

67. Benjamini Y, Hochberg Y. Controlling the false discovery rate: A practical and powerful approach to multiple testing. J R Stat Soc Series B Stat Methodol. 1995; 57:289-300.

68. Prinstein MJ, Nock MK, Spirito A, Grapentine WL. Multimethod assessment of suicidality in adolescent psychiatric inpatients: Preliminary results. J Am Acad Child Adolesc Psychiatry. 2001; 40:1053-1061. [PubMed: 11556629]

69. Weissman MM, Bland RC, Canino GJ, Greenwald S, Hwu HG, Joyce PR, et al. Prevalence of suicide ideation and suicide attempts in nine countries. Psychol Med. 1999; 29:9-17. [PubMed: 10077289]

70. Favaro A, Caregaro L, Di Pascoli L, Brambilla F, Santonastaso P. Total serum cholesterol and suicidality in anorexia nervosa. Psychosom Med. 2004; 66:548-552. [PubMed: 15272101]

71. Favaro A, Santonastaso P. Suicidality in eating disorders: Clinical and psychological correlates. Acta Psychiat Scand. 1997; 95:508-514. [PubMed: 9242846]

72. Youssef G, Plancherel B, Laget J, Corcos M, Flament MF, Halfon O. Personality trait risk factors for attempted suicide among young women with eating disorders. Eur Psychiatry. 2004; 19:131139. [PubMed: 15158919]

73. Björck C, Clinton D, Sohlberg S, Hällström, Norring C. Interpersonal profiles in eating disorders: Ratings of SASB self-image. Psychol Psychother. 2003; 76:337-349. [PubMed: 14670185]

74. Brevard A, Lester D, Yang BJ. A comparison of suicide notes written by suicide completers and suicide attempters. Crisis. 1990; 11:7-11. [PubMed: 2376149]

75. Mikolajczak M, Petrides KV, Hurry J. Adolescents choosing self-harm as an emotion regulation strategy: The protective role of trait emotional intelligence. Brit J Clin Psych. 2009; 48:181-193.

76. Foulon C, Guelfi JD, Kipman A, Adès J, Romo L, Houdeyer K, et al. Switching to the bingeing/ purging subtype of anorexia nervosa is frequently associated with suicidal attempts. Eur Psychiatry. 2007; 22:513-519. [PubMed: 17482799] 


\section{Table 1}

$\mathrm{N}(\%)$ of patients who attempted suicide at any time, prior to registration into Stepwise, and after registration by eating disorder diagnosis

\begin{tabular}{lcrcrrr}
\hline & \multicolumn{2}{l}{ Suicide attempt any time } & \multicolumn{2}{c}{ Suicide attempt prior to registration } & \multicolumn{2}{c}{ Suicide attempt after registration } \\
\cline { 2 - 6 } & \multicolumn{1}{c}{ No } & Yes & \multicolumn{1}{c}{ No } & Yes & No & Yes \\
\hline ANR & $423(91.4)$ & $40(8.6)$ & $440(95.0)$ & $23(5.0)$ & $443(95.7)$ & $20(4.3)$ \\
ANBP & $109(85.2)$ & $19(14.8)$ & $118(92.2)$ & $10(7.8)$ & $115(89.8)$ & $13(10.2)$ \\
BN & $595(88.7)$ & $76(11.3)$ & $612(91.2)$ & $59(8.8)$ & $645(96.1)$ & $26(3.9)$ \\
BED & $125(93.3)$ & $9(6.7)$ & $126(94.0)$ & $8(6.0)$ & $131(97.8)$ & $3(2.2)$ \\
EDNOS & $790(92.7)$ & $62(7.3)$ & $812(95.3)$ & $40(4.7)$ & $828(97.2)$ & $24(2.8)$ \\
\hline
\end{tabular}

Note . Stepwise $=$ quality assurance database of specialized eating disorders care in Sweden (patients are registered into Stepwise once intent to treat is established); $\mathrm{ANR}=$ anorexia nervosa restricting type; $\mathrm{ANBP}=$ anorexia nervosa binge-eating/purging; $\mathrm{BN}=$ bulimia nervosa; $\mathrm{BED}=$ binge eating disorder; EDNOS = eating disorder not otherwise specified. 


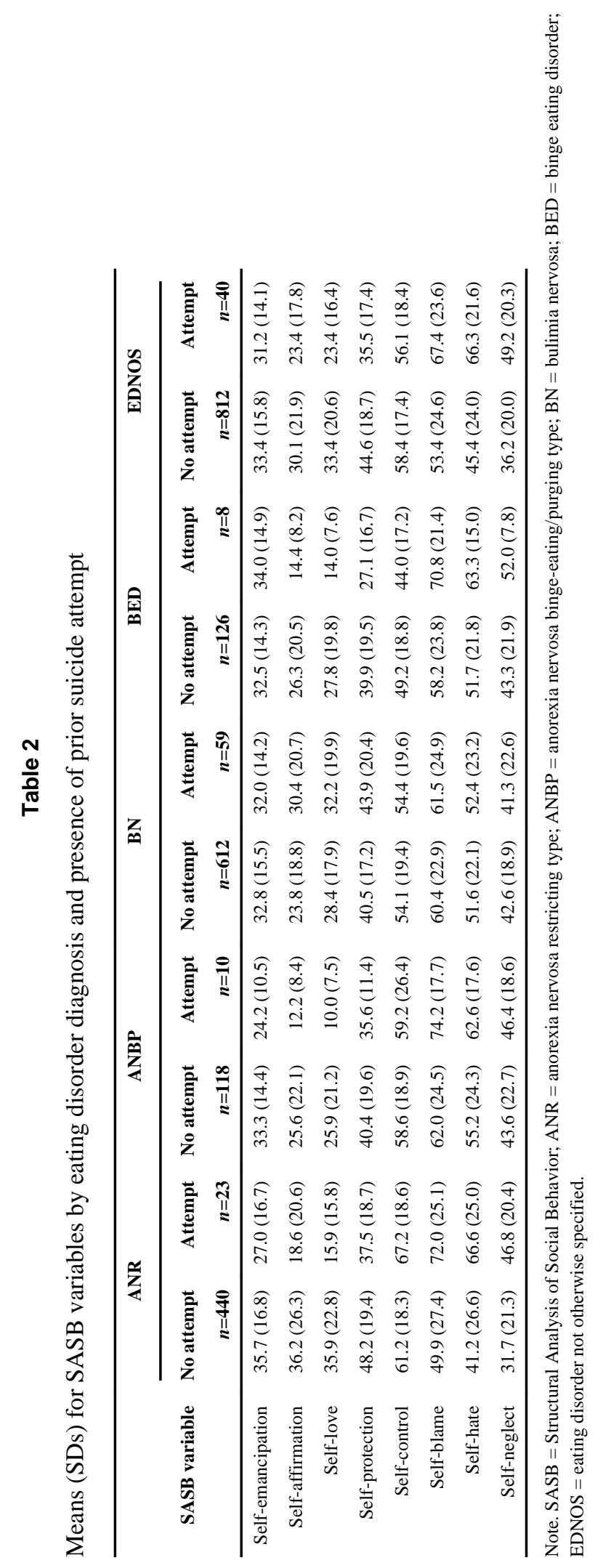

Compr Psychiatry. Author manuscript; available in PMC 2015 April 01. 


\section{Table 3}

Means (SDs) for SASB variables by eating disorder diagnosis and presence of suicide attempts/completions after registration into Stepwise

\begin{tabular}{rrrrrrrrr}
\hline & \multicolumn{2}{c}{ ANR } & \multicolumn{2}{c}{ ANBP } & \multicolumn{2}{c}{ BN } & \multicolumn{2}{c}{ EDNOS } \\
\cline { 2 - 10 } SASB variable & No attempt & Attempt & No attempt & Attempt & No attempt & Attempt & No attempt & Attempt \\
& $\mathbf{n = 4 4 3}$ & $\boldsymbol{n = 2 0}$ & $\boldsymbol{n = 1 1 5}$ & $\boldsymbol{n = 1 3}$ & $\boldsymbol{n = 6 4 5}$ & $\boldsymbol{n = 2 6}$ & $\boldsymbol{n = 8 2 8}$ & $\boldsymbol{n = 2 4}$ \\
\hline Self-emancipation & $35.5(16.8)$ & $30.3(18.2)$ & $32.6(14.5)$ & $33.1(13.0)$ & $32.9(15.4)$ & $27.2(13.2)$ & $33.1(15.8)$ & $38.8(13.2)$ \\
Self-affirmation & $35.6(26.3)$ & $29.3(26.8)$ & $25.5(22.4)$ & $15.8(10.3)$ & $24.8(19.1)$ & $13.2(12.4)$ & $29.7(21.9)$ & $30.0(17.0)$ \\
Self-love & $35.5(22.9)$ & $23.1(21.3)$ & $25.7(21.4)$ & $15.4(12.4)$ & $29.1(18.1)$ & $18.0(13.5)$ & $33.0(20.6)$ & $31.9(17.9)$ \\
Self-protection & $47.8(19.4)$ & $45.6(22.1)$ & $40.4(19.8)$ & $36.4(11.5)$ & $40.9(17.4)$ & $36.8(19.1)$ & $44.3(18.7)$ & $40.9(18.1)$ \\
Self-control & $61.2(18.5)$ & $69.7(14.3)$ & $59.0(19.3)$ & $56.0(21.2)$ & $54.4(19.3)$ & $48.5(21.1)$ & $58.3(17.5)$ & $55.8(16.5)$ \\
Self-blame & $50.6(27.6)$ & $59.3(30.8)$ & $61.8(24.9)$ & $72.8(14.0)$ & $60.0(23.1)$ & $74.4(18.4)$ & $54.1(24.7)$ & $52.0(24.3)$ \\
Self-hate & $42.0(26.8)$ & $54.0(32.1)$ & $55.0(24.4)$ & $62.5(18.1)$ & $51.1(22.1)$ & $67.7(17.1)$ & $46.1(24.3)$ & $54.7(20.4)$ \\
Self-neglect & $32.1(21.1)$ & $41.2(27.7)$ & $42.8(22.7)$ & $52.8(17.7)$ & $42.0(18.9)$ & $54.7(23.8)$ & $36.7(20.2)$ & $42.2(18.9)$ \\
\hline
\end{tabular}

Note. SASB = Structural Analysis of Social Behavior; Stepwise = quality assurance database of specialized eating disorders care in Sweden (patients are registered into Stepwise once intent totreat is established); ANR = anorexia nervosa restricting type; ANBP = anorexia nervosa bingeeating/purging; $\mathrm{BN}=$ bulimia nervosa; EDNOS = eating disorder not otherwise specified. 


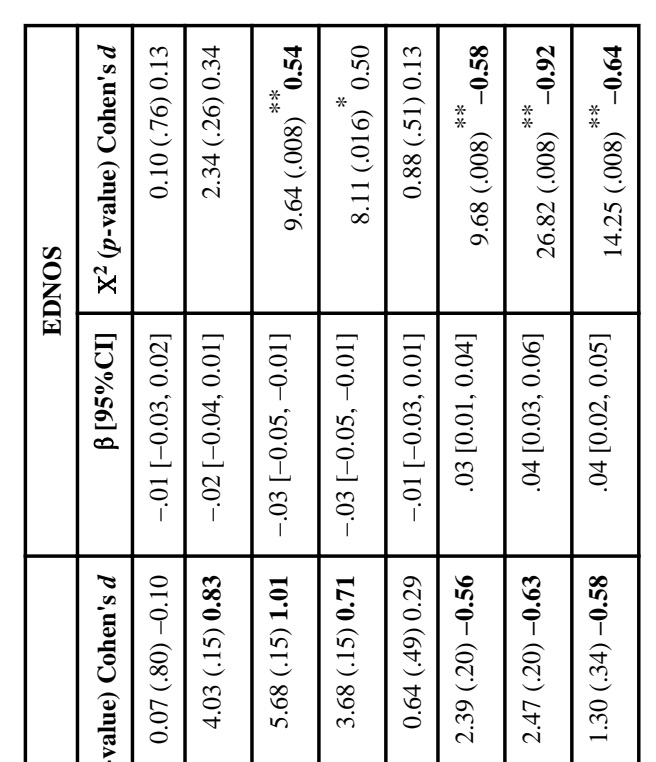

黄

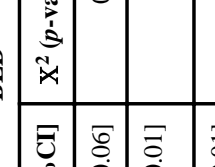

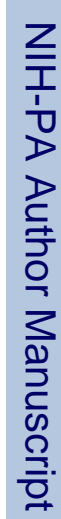

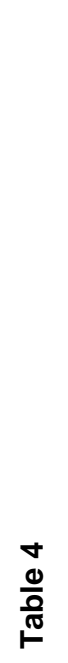

Z

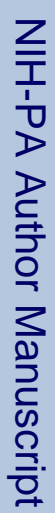

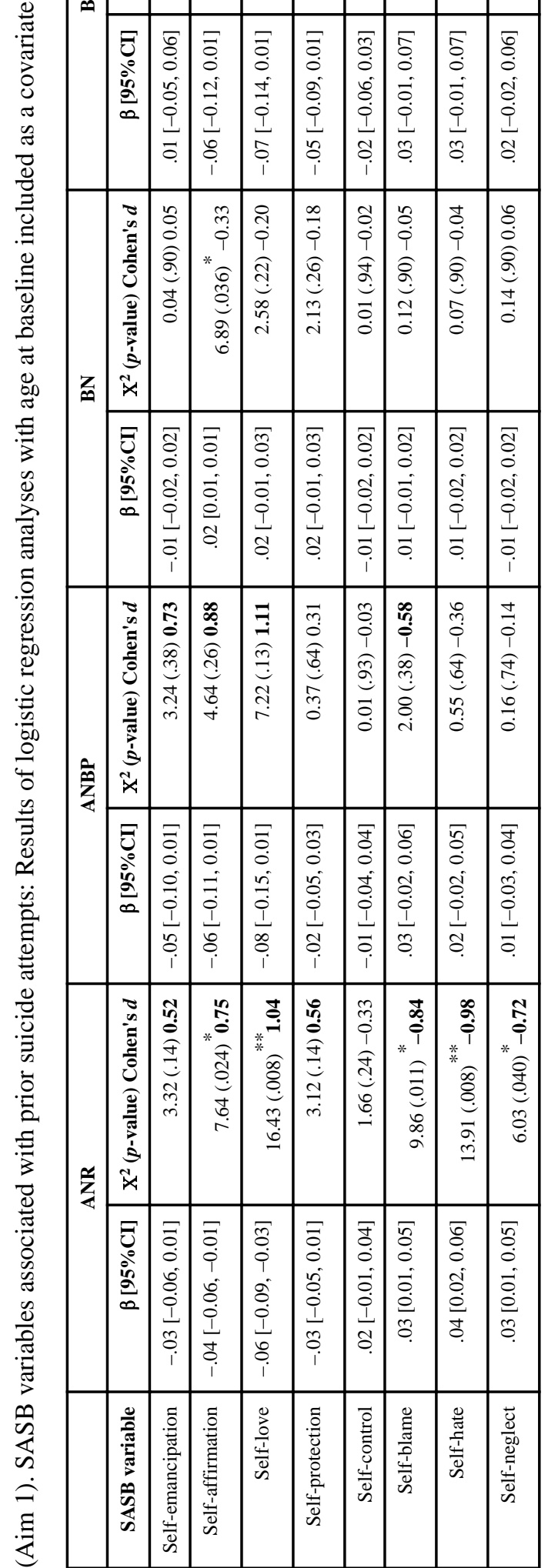

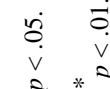




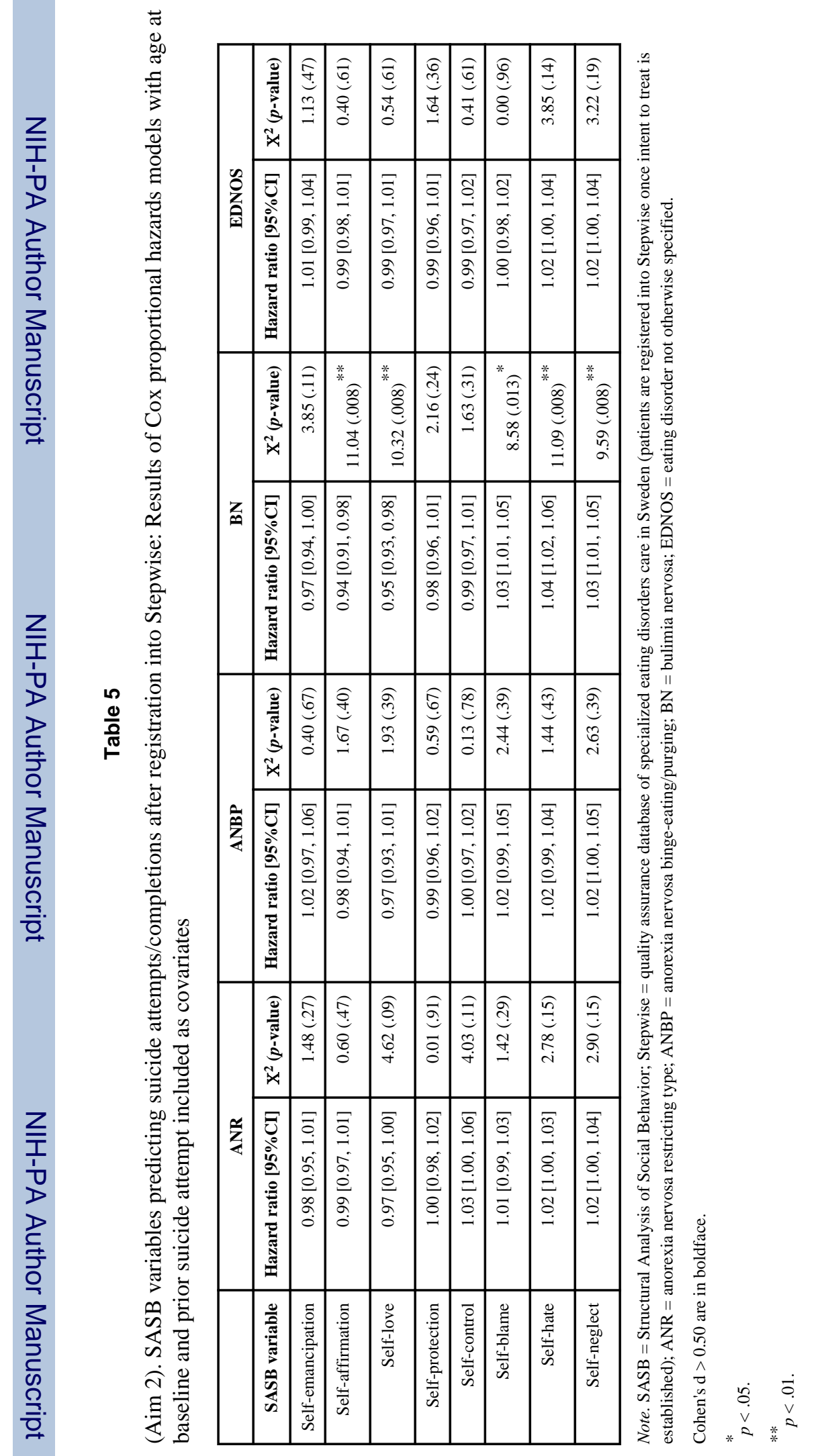

